\title{
Two-loop renormalization of fermion bilinears on the lattice
}

\section{A. Skouroupathis ${ }^{* \dagger}$ and H. Panagopoulos}

University of Cyprus, Department of Physics

E-mail: php4as01@ucy.ac.cy, haris@ucy.ac.cy

We compute the renormalization functions on the lattice, in the $R I^{\prime}$ scheme, of local bilinear quark operators $\bar{\psi} \Gamma \psi$, where $\Gamma=\hat{1}, \gamma_{5}, \gamma_{\mu}, \gamma_{5} \gamma_{\mu}, \gamma_{5} \sigma_{\mu \nu}$. This calculation is carried out to two loops for the first time. We consider both the flavor non-singlet and singlet operators.

As a prerequisite for the above, we compute the quark field renormalization, $Z_{\psi}^{L, R I^{\prime}}$, up to two loops. We also compute the 1-loop renormalization functions for the gluon field, $Z_{A}^{L, R I^{\prime}}$, ghost field, $Z_{c}^{L, R I^{\prime}}$, gauge parameter, $Z_{\alpha}^{L, R I^{\prime}}$, and coupling constant $Z_{c}^{L, R I^{\prime}}$.

We use the clover action for fermions and the Wilson action for gluons. Our results are given as an explicit function of the coupling constant $a_{\circ}=g_{\circ}^{2} / 16 \pi^{2}$, the clover coefficient $c_{S W}$, and the number of fermion colors $\left(N_{c}\right)$ and flavors $\left(N_{f}\right)$, in the renormalized Feynman gauge. All 1-loop quantities are evaluated in an arbitrary gauge.

Finally, we present our results in the $\overline{M S}$ scheme, for easier comparison with calculations in the continuum $[1,2]$. We have generalized to fermionic fields in an arbitrary representation. Some special features of superficially divergent integrals, obtained from the evaluation of twoloop Feynman diagrams (a partial set of such integrals can be found in Ref. [3]), are presented in detail in Ref. [4].

The 2009 Europhysics Conference on High Energy Physics,

July 16 - 22, 2009

Krakow, Poland

\footnotetext{
* Speaker.

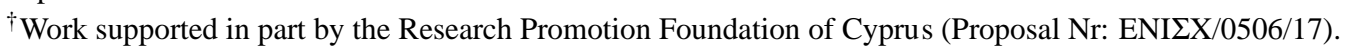




\section{Introduction}

Numerical simulations of QCD, formulated on the lattice, make use of a variety of composite operators, made out of quark fields. A proper renormalization of these operators is essential for the extraction of physical results from the dimensionless quantities measured in numerical simulations.

The main results presented in this work are the lattice 2-loop bare Green's functions (amputated, $1 \mathrm{PI}), \Sigma_{\Gamma}^{L}\left(q, a_{\mathrm{L}}\right)$, and the corresponding 2-loop renormalization functions, $Z_{\Gamma}^{L, R I^{\prime}}$ and $Z_{\Gamma}^{L, \overline{M S}}$.

\section{The $\mathbf{R I}^{\prime}$ renormalization scheme}

We define renormalized operators by: $\mathscr{O}_{\Gamma}^{R I^{\prime}}=Z_{\Gamma}^{L, R I^{\prime}}\left(a_{\mathrm{L}} \bar{\mu}\right) \mathscr{O}_{\Gamma \circ}$. In order to extract the 2-loop renormalization functions, we employ the $R I^{\prime}$ renormalization conditions:

$$
\lim _{a_{\mathrm{L}} \rightarrow 0}\left[Z_{\psi}^{L, R I^{\prime}} Z_{\Gamma}^{L, R I^{\prime}} \Sigma_{\Gamma}^{L}\left(q a_{\mathrm{L}}\right)\right]_{q^{2}=\bar{\mu}^{2}}=\Gamma_{\text {tree }},
$$

where $\Sigma_{\Gamma}^{L}\left(q a_{\mathrm{L}}\right)$ is the appropriate bare 1PI 2-point Green's function on the lattice and $\Gamma_{\text {tree }}$ is its tree-level value. For the conversion of results in the $\overline{M S}$ scheme, see Refs. [4, 5].

\section{Results}

We present the expression obtained from the computation of Feynman diagrams, in the case of the vector renormalization function $\left(c_{F} \equiv\left(N_{c}^{2}-1\right) /\left(2 N_{c}\right)\right)$. For our complete set of results, see Refs. $[4,5]$.

$$
\begin{aligned}
Z_{V}^{L, R I^{\prime}}=1 & +a_{\circ} c_{F}\left[-20.617798655(6)+4.745564682(3) c_{\mathrm{SW}}+0.543168028(5) c_{\mathrm{SW}}^{2}\right] \\
+ & a_{\circ}^{2} c_{F}\left[N_{f}\left(25.610(3)-11.058(1) c_{\mathrm{SW}}+33.937(3) c_{\mathrm{SW}}^{2}-13.5286(6) c_{\mathrm{SW}}^{3}-1.2914(6) c_{\mathrm{SW}}^{4}\right)\right. \\
& +c_{F}\left(-539.78(1)-223.57(2) c_{\mathrm{SW}}-104.116(5) c_{\mathrm{SW}}^{2}-32.2623(8) c_{\mathrm{SW}}^{3}+4.5575(3) c_{\mathrm{SW}}^{4}\right) \\
& \left.+N_{c}\left(-51.59(1)+18.543(5) c_{\mathrm{SW}}+20.960(6) c_{\mathrm{SW}}^{2}+2.5121(5) c_{\mathrm{SW}}^{3}+0.1765(1) c_{\mathrm{SW}}^{4}\right)\right]
\end{aligned}
$$

\section{References}

[1] J.A. Gracey, Three loop anomalous dimension of non-singlet quark currents in the RI' scheme, Nucl. Phys B662 (2003) 247 [hep-ph/ 0304113 ].

[2] S.A. Larin and J.A.M. Vermaseren, The three-loop beta-fubction and anomalous diensions, Phys. Lett. B303 (1993) 334 [hep-ph/9302208]; S.A. Larin, The renormalization of the axial anomaly in dimensional regularization, Phys. Lett. B303 (1993) 113 [hep-ph/9302240].

[3] M. Lüscher and P. Weisz, Computation of the relation between the bare lattice coupling and the $\overline{M S}$ coupling in the $S U(N)$ gauge theories to two loops, Phys. Lett. B349 (1995) 165 [hep-lat/9502001]; Nucl. Phys. B452 (1995) 234 [hep-lat/9505011].

[4] A. Skouroupathis and H. Panagopoulos, Two-loop renormalization of vector, axial-vector and tensor fermion bilinears on the lattice, Phys. Rev. D79 (2009) 094508 [arXiv: 0811 . 4264].

[5] A. Skouroupathis and H. Panagopoulos, Two-loop renormalization of scalar and pseudoscalar fermion bilinears on the lattice, Phys. Rev. D76 (2007) 094514; Erratum-ibid. D78 (2008) 119901 [arXiv:0707.2906]. 